\title{
The Advantage of a Total Knee Arthroplasty with Rotating Platform is Only Theoretical: Prospective Analysis of 1,152 Arthroplasties
}

\author{
H.P. Delport*
}

Department of Orthopaedic Surgery, AZ Nikolaas, SintNiklaas, Belgium

\begin{abstract}
Background: The aim of total knee surgery is to provide patients with end-stage osteoarthritis of the knee with both pain relief and a functional range of motion with a securely fixed prosthesis for the long term. Many types of implants are designed to achieve these goals. Only clinical outcome studies are able to substantiate the superiority of one design over another. Our primary research question was to determine whether patients receiving a rotating platform implant had a better functional outcome.
\end{abstract}

Methods: A total of 1,152 Performance Total Knee Arthroplastiesin 943 patients were studied prospectively. In 561 cases, the cruciate retaining model (CR) was used. In 591 cases the cruciate substituting with posterior-stabilized model (PS) was implanted (324 cases with a fixed bearing (PSFB) and 267 cases with a rotating platform (PSRP)).

Results: The Clinical KSS score was similar for the three types at 1, 5, 10 and 15 years post-operatively. The Functional score also remained similar for all types until 10 years; at 15 years functional results of the CR group decreased.

Conclusions: Neither clinically nor radiographically did the use of a rotating platform prove to be more advantageous than fixed bearing tibial components. Thus, the advantage of a Total Knee Arthroplasty with Rotating Platform remains theoretical.

Keywords: Total knee arthroplasty, rotating platform, clinical outcome.

\section{INTRODUCTION}

Performing long-term follow-up studies of a particular type of implant is not a simple matter in orthopedics. Many designs of implant devices produced by orthopedic companies are modified after being used for a less than a decade. Usually, new types of these devices claim improvement compared to the older ones. However, in choosing the most appropriate implant for his or her patient, the orthopedic surgeon must be aware of the possibility of a market drive with the introduction of new and "better" products.

Total knee arthroplasty (TKA) is very effective for the treatment of severe osteoarthritis of the knee joint. Many good to excellent results have been reported. Nevertheless, surgeons continue to modify the knee prosthesis design in an attempt to improve the results. Therefore, mobile bearing tibial components of different designs were introduced. Theoretically they were meant to reduce wear [1]; thus, increasing longevity. They also were designed to improve function by attempting to make the kinematics more similar to the native knee [2].

The author began using the Performance TKA (Performance, BIOMET Spain Orthopaedics, S.L. Calle Islas Baleares, 50, 46988 Fuente del Jarro, Valencia, SPAIN) in 1989; he initially compared it to other TKA designs on a 50/50 basis. Due to the excellent results, headvanced to

*Address correspondence to this author at the Department of Orthopaedic Surgery, AZ Nikolaas, SintNiklaas, Belgium; Tel: 0032 33690230;

E-mail: hendrik.delport@ortho-expert.be
100\% use of the Performance TKA in 1995 and decided to begin a prospective investigation. Due to the easy access of patients to orthopaedic healthcare and the short distance between the providing hospitals, it is very difficult to verify correct follow-up. Strong competition influences indication and decision for revision surgery in our community, preventing a single surgeon from performing a genuine survival analysis. The hypothesis, however, was to determine whether patients receiving a rotating platform implant had a better functional outcome than those who received the fixed bearing designs.

\section{PATIENTS AND METHODS}

From 1995 through 2007, we conducted a prospective observational study of 1,152 TKA by a nonrandomized method. A final follow-up was done in 2012. This type of knee arthroplasty became available in three different models. The cruciate retaining (CR) model and the posterior stabilized (PS) model, both with a fixed tibial polyethylene bearing, were used initially. The PS with a rotating platform (PSRP) was introduced in 1992. After an initial evaluation period, the study was initiated in 1995 and concluded in 2007, with data collection until 2012.

Initially the study was meant to compare two types, namely the CR and the PS fixed bearing. Due to possible theoretical advantages, many orthopedic companies developed total knee implants with a mobile bearing. In the case of the Performance TKA, the company Biomet choose to design a TKA, which was posterior-stabilized (PS) and had a rotating platform (RP). We included this new type in our prospective study. Therefore this study is not a 
randomized, controlled trial, but rather a historical prospective investigation by a single surgeon using three different versions of a single implant.

The indications for performing TKA were (1) disabling knee pain and functional loss, unresolved with non-operative treatment; (2) radiographic evidence of advanced arthritic changes; and (3) with all different forms of varus, valgus, and flexion contracture deformities. The contraindications were the presence of active infection or neuropathic disorders.

This study deals with three cohorts that are followed forward prospectively and then observed for outcome. The participants of the groups are classified according to the intervention they received. The comparison group is the group of patients from the same population: our clinical practice patients who did receive the other type of implant. We choose an objective outcome to be revision or removal of the implant.

All patients were operated on by the same surgeon (HPD), using the same knee replacement system but using three different configurations: (1) fixed PCL-retaining (CR), (2)fixed posterior-stabilized (PSFB), and (3) rotating platform posterior-stabilized (PSRP). The Performance design allows the use of two different femoral components, one for the PCL-retaining configuration and the other for the fixed or rotating platform posterior-stabilized configurations. Both the PCL-retaining and posterior-stabilized femoral components, and their corresponding bearings, have the same sagittal radius, but differ in the coronal plane. The posterior-stabilized coronal plane condylar radius is smaller, resulting in a more rounded femoral component, while the mobile posterior-stabilized bearing is more concave in both the sagittal and coronal planes.

The distribution of the three different configurations of tibial bearing was as follows: fixed-bearing PCL-retaining (561 patients), posterior stabilized or cruciate substituting (591 patients, 324 fixed-bearing posterior-stabilized and 267 rotating platform posterior-stabilized). For the fixed-bearing PCL-retaining knee replacements, a posterior-lipped insert was used. Fixed and mobile-bearing posterior-stabilized knee replacements had similar cam-post mechanisms. Comparing equal sizes of component in the femur and tibia, the contact point between a posterior-stabilized femoral component and insert at the cam-post junction theoretically occurs at $30^{\circ}$ of flexion in the fixed and $40^{\circ}$ in the rotating platform configuration. This is the result of the interactive effect of both the antero-posterior position of the post and the design of the bearing in the sagittal plane. Both PCLretaining and posterior-stabilized fixed-bearings are secured to the tibial tray through an anterior slot, a peripheral rim and a central locking screw, whereas a posterior-stabilized rotating platform insert can freely rotate around a central peg on a polished tibial tray. The underside of the tray and the tibial stem are identical in both the fixed and mobile designs of tibial tray. Only patients with a pre-operative diagnosis of end-stage osteoarthritis of the knee were included in the study at a minimum follow-up of 3 months (mean $60 \mathrm{~m}$., range 3-204 $\mathrm{m}$ ).

We prospectively followed 1152 knees (943 patients)who underwent a Performance Total Knee Replacement. Of these patients, there were 701 women $(74.34 \%)$ and 242 men (25.66\%); the women had an average age of 71 years (range:35-92 years); the men had an average age of 69.7 years (range:31-96 years). At the time of surgery the average BMI was $27 \mathrm{Kg} / \mathrm{m}^{2}$.

The minimum follow-up was 6 months and the mean follow-up was 60 months (range 3-204 months). An institutional review board approved the analysis.

\section{Surgical Technique}

The majority of the patients $(90 \%)$ received spinal anesthesia; $10 \%$ received general anesthesia. The author performs a medial subvastus approach whenever possible (95\%). Obesity, contractures, and deformity were not contraindications. The patient is positioned supine in the standard fashion. We initially employed a post to support the foot, so that the knee can be flexed and maintained at 90 degrees of flexion. Later this post was replaced by a foot holding device. The skin incision was made directly midline or slightly medial to midline. The length of the incision is based on the underlying pathology. Generally, it extends from the superior pole of the patella to the inferior aspect of the tibial tubercle. The incision is advanced sharply through the subcutaneous tissue down to the retinacular tissue. Fullthickness medial and lateral flaps are then created sharply with meticulous hemostasis. This creation of a large, fullthickness flaps is important because it allows the extensor mechanism to be mobilized deep to the subcutaneous tissues. It also prevents skin flap necrosis.

The distal insertion of the vastus medialis obliquus (VMO) on the patella is exposed. The fascia overlying the VMO is released sharply, taking care not to injure any underlying muscle fibers. An oblique capsular incision is then made just distal to the VMO, beginning posteriorly at the level of the intermuscular septum and extending laterally, parallel to the inferior border of the muscle, towards the medial border of the patella.

At the medial border of the patella, the arthrotomy is extended distally, taking care to leave a cuff of tissue attached to the patella for closure. The arthrotomy incision is carried distally across the joint line and parallel to the medial border of the patellar tendon. Care is taken to leave a remnant of the medial meniscus to find the joint line in case a revision procedure is necessary later.

A standard closure is performed at the end of the case, usually in $75^{\circ}$ of flexion. The closure is quite simple as no muscle needs to be repaired. We have found the subvastus approach to provide excellent exposure that facilitates advancement of rehabilitation after knee surgery. There is almost no need for lateral retinaculum release. Postoperative bleeding ceases promptly, due to the complete sealing of the joint. Notwithstanding the hype of minimal invasive surgery (MIS), we place less emphasis on the length of the skin incision and more on careful dissection of large medial and lateral skin flaps and atraumatic mobilization of the muscle.

The author performed all TKAs using three different types of the same brand of TKA with the use of the previously described technique [3], using standard alignment instrumentation. Medial and lateral tibial and femoral osteophytes were removed. In the CR version, the posterior 
cruciate ligament was left intact. Posterior osteophytes were removed and in case of flexion contracture a posterior capsular release was performed. The patella was resurfaced in $92 \%$ of the cases.

Patellar tracking and ligament balance were evaluated clinically by the surgeons feel. A release of the collateral ligaments, the posterior cruciate for the CR version, and the retinaculum was performed very meticulously to obtain a balanced TKA. This correction was always based on a personal evaluation of the experienced surgeon without additional balancing instruments. Femoral components were cemented in $98 \%$ of the cases. The tibial component was cemented in $95 \%$ of cases.

For anticoagulant therapy we used enoxyparine for 6 weeks beginning the day after surgery. Postoperatively the patients initially began ambulating on the third day; subsequently, however, ambulation with a walker or canes was begun on the first postoperative day. The patients were discharged home or to a nursing facility as soon as they were confident that they could walk independently; further physiotherapy was conducted following discharge. On the day of discharge they underwent an AP non-weight-bearing $\mathrm{x}$-ray.

\section{Clinical Evaluation}

We prospectively acquired preoperative and postoperative data. Reoperation for any reason including stiffness, instability, wear, loosening, fracture, infection, or hematoma were recorded. The patients were assessed by a physical examination and knee scoring pre-operatively, at six weeks, at one year after surgery, and annually thereafter. Not all patients were disciplined enough to return each year. We used the Knee Society score [4] to determine the Knee and Functional scores pre-operatively and at each visit. This was recorded using the Orthowave software system (OW; Copyright $(\odot 2009$ Aria Software) as designed by Jean-Alain Epinette (200, Rue d'Auvergne, 62700 Bruay la Buissière, France). Knee rating and function were separately assessed. The maximum knee score was 100 points with three parameters: pain, stability, and range of motion. Flexion contracture, extension lag, and misalignment are dealt with as deductions. The maximum functional score was also 100 points; it considers walking distance and stair climbing, with deductions for walking aids.

\section{Radiologic Evaluation}

Radiographs were obtained pre-operatively, at six weeks, at one year post-operatively, and on return visits thereafter. Standard antero-posterior (AP) and lateral views of the knee joint were taken. A CT scan was only performed in preparation for a revision (if necessary). Pre-operative and post-operative overall alignment, as well as tibial component alignment were evaluated by the study nurse (I.M.). Postoperative AP knee radiographs were used to measure component alignment. Tibial alignment was measured between a line parallel to the baseplate of the component and a line along the tibial shaft axis. Overall femoro-tibial angle was measured between a line along the femoral shaft axis and the tibial shaft axis with results less than $0^{\circ}$ indicating valgus alignment. Osteolysis was noted as $<1 \mathrm{~mm}$ or progressive, according to the Knee Society scoring method [5].

\section{Statistical Analysis of the Follow-Up Data}

These data are statistically analyzed using one way anova followed by unpaired students t-tests, interpreting Bonferoni correction. Frequency data was judged using chi-squared tests; statistical significance was set at 0.05 .

These data are calculated as percent changes against initial value and data are obtained at 1, 6, 12 months, and yearly thereafter for a maximum of 17 years. Statistical analysis is done on data until year 10 because of the limited numbers available beyond this time point. The mixed procedure is the test of first choice because it corrects for missing values and has two main goals: analyzing the time element and simultaneously the combination of time and group.

\section{RESULTS}

We prospectively analyzed a cohort of 1,152 patients receiving a total knee arthroplasty (TKA) using the Performance Total Knee Prosthesis. The study group was homogeneous for age $(P<0.001)$, but not for gender $\left(\mathrm{X}^{2}\right.$ $=0.25(\mathrm{NS}))$. Preoperative values for the Function-scores are equal in the 3 groups: $\mathrm{F}=0.57$ (NS). The original number of patients was 943 and the number of knees studied was 1,152 (267 were PSRPs). The number of patients who died was: 22 patients (2.3\%; CR: $2.4 \%$; PSFB: $0.6 \%$; PSRP: $4.8 \%$ ). The percentage of patients who were lost to follow-upwas $10.34 \%$. Among the patients/knees currently being studied (591 PSs), 267 were RPs and 324 were FBs.

\section{Knee and Functional Scores}

The average preoperative subjective and functional Knee Society scores were 33 and 42 for patients with a CR type implant, 32 and 42 for the patients with a fixed bearing PS implant, and 38 and 42 for the patients with a rotating platform type PS implant (Tables $\mathbf{1}$ and 2).

Table 1. Clinical KS Score

\begin{tabular}{|c|c|c|c|c|c|}
\hline Clinical KSS Score & Pre-op & $\mathbf{1} \mathbf{y}$ & $\mathbf{5 y}$ & $\mathbf{1 0 y}$ & $\mathbf{1 5 y}$ \\
\hline \hline CR & 33 & 97 & 98 & 98 & 100 \\
\hline PSFB & 32 & 96 & 96 & 99 & 96 \\
\hline PSRP & 38 & 97 & 99 & 99 & 100 \\
\hline
\end{tabular}

Table 2. Functional KS Score

\begin{tabular}{|c|c|c|c|c|c|}
\hline Functional KSS Score & Pre-op & $\mathbf{1} \mathbf{y}$ & $\mathbf{5 y}$ & $\mathbf{1 0 y}$ & $\mathbf{1 5 y}$ \\
\hline \hline CR & 42 & 93 & 94 & 93 & 75 \\
\hline PSFB & 42 & 93 & 97 & 89 & 90 \\
\hline PSRP & 42 & 92 & 97 & 95 & 91 \\
\hline
\end{tabular}

The mean pre- and post-operative Knee Society knee and functional scores showed no statistically difference between the groups (Student's t-test $P=0$ ). 


\section{Radiologic Findings}

The number of patients with available radiographs was 677.

Table 3. Radiologic Alignment

\begin{tabular}{|c|c|c|c|c|}
\hline & All TKA & CR & PSFB & PSRP \\
\hline \hline F/T angle & $-1.82^{\circ}$ & $-1.96^{\circ}$ & $-1.78^{\circ}$ & $-1.76^{\circ}$ \\
\hline Tibial angle & $0.75^{\circ}$ & $0.64^{\circ}$ & $1.0^{\circ}$ & $0.73^{\circ}$ \\
\hline
\end{tabular}

The radiographic assessment showed that the overall post-operative femoro-tibial $(\mathrm{F} / \mathrm{T})$ angle was almost always in a slight valgus of $-1.82^{\circ}\left(+2.6^{\circ}\right.$ to $\left.-7.4^{\circ}\right)$ with very few outliers. However, the tibial component was positioned in a minimal varus of $0.75^{\circ}\left(+3.4^{\circ}\right.$ to $\left.-2.5^{\circ}\right)$ (Table 3). Osteolysis of less than $1 \mathrm{~mm}$ was noted primarily in zone 1 (under the medial tibial plateau) in $34 \%$ of the cases in all three groups. Only 3 patients in the PSRP group, 4 in the PSFB group, and 6 in the CR group showed progressive signs of osteolysis.

The percentage of arthroplasties revised for any reason (aseptic loosening, osteolysis, component breakage, instability, or infection) was $1.2 \%$. The revised arthroplasties included in the current series, reported separately are: tibial failure $1.0 \%$, femoral failure $0.2 \%$, infection $0.08 \%$. Survivorship analysis was not possible due to the local culture of healthcare shopping, making exact revision numbers unavailable (Table 4).

Table 4. Overview of Failures and Deaths

\begin{tabular}{|c|c|c|c|c|}
\hline & Total TKA & CR & PSFB & PSRP \\
\hline \hline \% dead & 2.3 & 2.4 & 0.6 & 4.8 \\
\hline \% Tibial failure & 1.0 & 1.7 & 0.6 & 0.0 \\
\hline \% Femoral failure & 0.2 & 0.5 & 0.0 & 0.0 \\
\hline \% infection & 0.08 & 0.0 & 0.3 & 0.0 \\
\hline \% retrieval & 1.2 & 1.9 & 0.9 & 0.0 \\
\hline
\end{tabular}

\section{DISCUSSION}

Long-term outcome and prognosis are particularly important in orthopaedic surgery because implants last many years. From our viewpoint as clinicians working in a community hospital setting, the prospective non-randomized cohort study was the only feasible design. During our study, the assignment of patients to the three groups was not at random. However the groups were well-matched, which limited the bias as much as possible. We have examined the time to revision or removal and the factors that possibly affect long-term survivorship. The main strength of this study is the large number of patients and the relatively long follow-up. However, the main limitation is the lack of completeness of both clinical and radiological evaluations. Many patients were lost to follow-up due to address change or a move to a senior facility.

When measuring clinical and functional scores, we could not find superiority for any of the bearing designs in our study. Comparing radiologic evaluations also did not reveal any difference. A number of other authors were also unable to find clinical or radiological difference, comparing fixed with mobile-bearing/rotating platform tibial components.

Only one study by C Hopley et al. [6], which reported on the survivorship of the LCS rotating platform TKA, appeared to have a higher survivorship at 10 years follow-up relative to generalizable reports from the Swedish Knee Registry. They also reported very high survivorship out to 20 years with a very low incidence of wear-related revision in the second decade.

Definite conclusions are impeded by the restriction of these kind of reviews to primarily observational data and the existence of several confounding factors that cannot not be controlled for. Nevertheless, in the subgroup of studies with at least 10 years of mean follow-up, some with up to 20 years, the number of revisions remained low, despite the fact that all these studies were comprised older grades of polyethylene with inferior wear properties. This suggests that the rate of revision for osteolysis, wear, and loosening remains low in the second decade after surgery. Many of the remaining revisions were accounted for by spinout of the tibial insert or instability; however, the overall incidence was low.

In our literature review, however, we counted 8,436 knees that were evaluated without finding any clinical or radiological difference between fixed-bearing and mobilebearing/rotating-platform TKA (Table 5).

\section{CONCLUSIONS}

In conclusion, our study found no evidence of superiority of the rotating platform over the fixed-bearing TKA. Since this was not a recruited study, the use of a CONSORT chart was, on a practical basis, not achievable or workable. We were only able to investigate the patients who returned spontaneously for follow-up or those who developed another orthopedic problem. The study nurse called the patients on a yearly basis and asked them to complete a questionnaire; however, she was unable to reach the entire patient group on a regular basis. With longer follow-up duration, the lost to follow-up group increased.

The major factors limiting the longevity of the prosthesis found at follow-up were not different for the three types. The concept that a rotating platform TKA is associated with a better clinical and radiological result than a fixed bearing TKA remains to be proven. Since this prosthesis is no longer available, and its successor, the Vanguard TKA, has no rotating platform, no determinations are applicable to the current successor. However, we conclude that a rotating platform definitely has theoretical advantages, but in clinical practice they are not obvious. It has been emphasized by many surgeons that good surgical technique is mandatory in a rotating platform TKA to avoid bearing dislocation and instability. It has also been commonly noted that a rotating platform TKA is contraindicated in cases of severe deformity. This was not experienced by the author. Therefore we hypothesize that other factors than design might be more important for improvement of function and patient satisfaction.

Neither clinically nor radiographically is the use of a rotating platform proven to advantageous over fixed bearing 
Table 5. Literature Comparing Outcome of Mobile-Bearing or Rotating Platform Versus Fixed Bearing TKAs

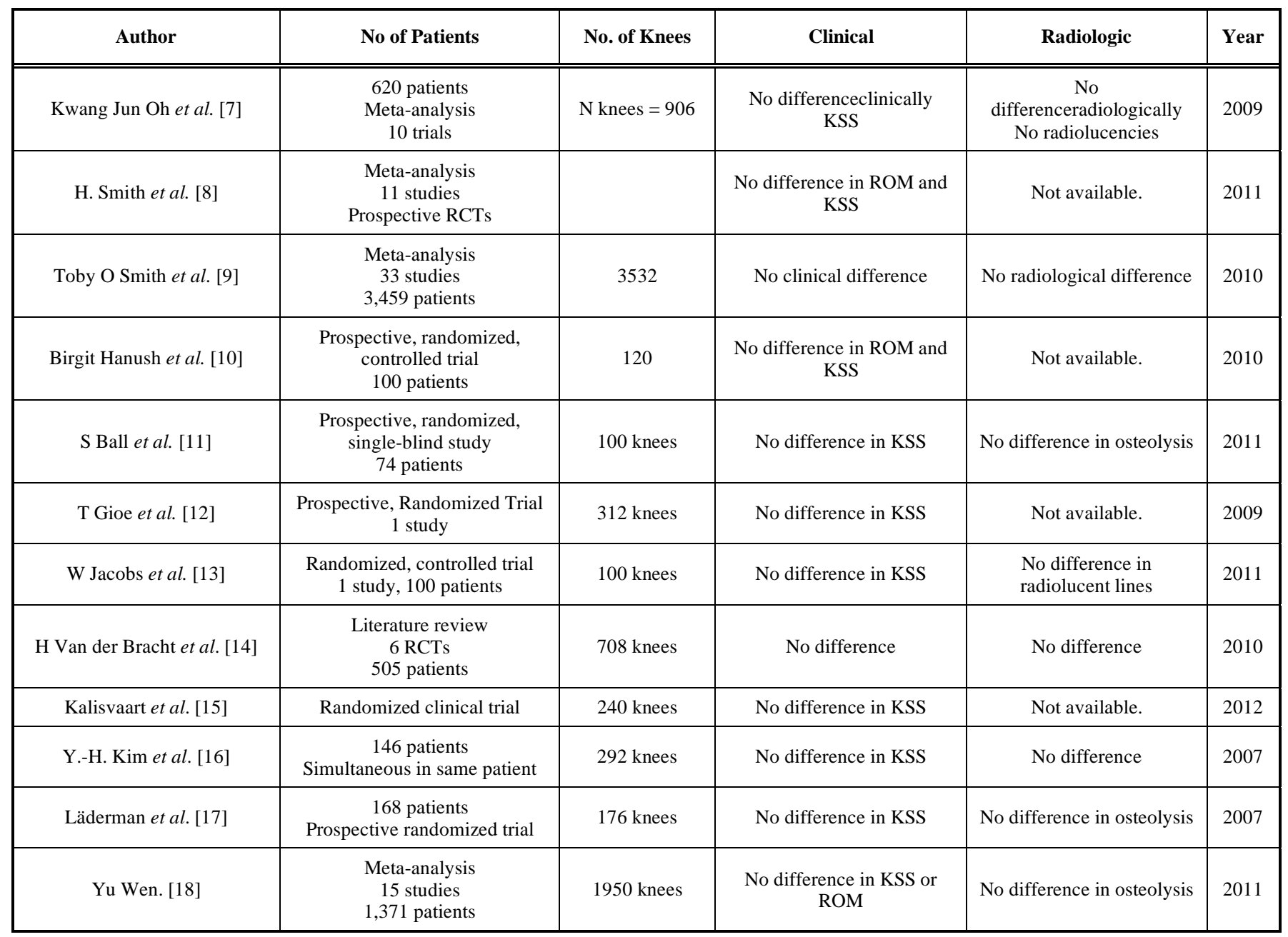

tibial components. The advantage of the Performance Total Knee Arthroplasty with Rotating Platform remains only theoretical. Our hypothesis was not confirmed. Other aspects, especially those concerning the surgical technique, must be investigated in search for improvement. Alignment accuracy and ligament balancing should be better defined through basic research. We believe that the relation between these surgery-dependent factors might guide us to a solution. Nevertheless, this evaluation is needed because we know that worldwide approximately $20 \%$ of TKA patients are less satisfied after their operation than they expected.

\section{CONFLICT OF INTEREST:}

The author confirms that this study has no conflict of interest.

\section{ACKNOWLEDGEMENTS}

The author would like to express many thanks to Ms. Ingrid Meyskens, research nurse, as well as Mr. J. Nijs. and Mr. T. Heylen for their statistical analysis.

\section{REFERENCES}

[1] Delport HP, Vander Sloten J, Bellemans J Comparative gravimetric Wear Analysis in mobile versus fixed-bearing posterior stabilized total knee prostheses. Acta Orthop Belg 2010; 76: 367-73.
[2] Delport HP, Banks S, De Schepper J and Bellemans J. A kinematic comparison of fixed- and mobile-bearing knee replacements.. $\mathrm{J}$ Bone Joint Surg Br 2006; 88: 1016-21.

[3] Bassett RW. Results of 1,000 Performance knees: cementless versus cemented fixation. J Arthropl 1998; 14(4): 409-13.

[4] Insall JN, Dorr LD, Scott RD, Scott WN. Rationale of the knee society clinical rating system. Clin Orthop 1989; 248: 13-4.

[5] Huang C-H, Ma H-M, Liau J-J, Ho F-Y, Cheng C-K. Osteolysis in failed total knee arthroplasty: a comparison of mobile-bearing and fixed-bearing knees. J Bone Joint Surg Am 2002; 84-A,12: 2224-9.

[6] Hopley CD, Crossett LS, Chen AF. Long-term clinical outcomes and survivorship after total knee arthroplasty using a rotating platform knee prosthesis. a meta-analysis. J Arthroplasty 2013; 28(1): 68-77.e1-3

[7] Oh KJ, Pandher DS, Lee SH, Sung Joon SD Jr, Lee ST. Metaanalysis comparing outcomes of fixed-bearing and mobile-bearing prostheses in total knee arthroplasty. J Arthroplasty 2009; 24(6): 873-84.

[8] Smith H, Jan M, Mahomed NN, Davey JR, Gandhi R. Metaanalysis and systematic review of clinical outcomes comparing mobile bearing and fixed bearing total knee arthroplasty. J Arthroplasty 2011; 26(8): 1205-13.

[9] Smith TO, Ejtehadi F, Nichols R, Davies L, Donell ST, Hing CB. Clinical and radiological outcomes of fixed- versus mobile-bearing total knee replacement: a meta-analysisKnee Surg Sports Traumatol Arthrosc 2010: 18(3): 325-40.

[10] Hanusch B, Lou TN, Warriner G, Hui A, Gregg P. Functional outcome of PFC Sigma fixed and rotating-platform total knee arthroplasty. A prospective randomised controlled trial. Int Orthop 2010; 34(3): 349-54. 
[11] Ball ST, Sanchez HB, Mahoney OM, Schmalzried TP. Fixed versus rotating platform total knee arthroplasty: a prospective, randomized, single-blind study. J Arthroplasty 2011; 26(4): 531-6.

[12] Gioe TJ, Glynn J, Sembrano J, Suthers K, Santos ER, Singh J. Mobile and fixed-bearing (all- polyethylene tibial component) total knee arthroplasty designs. A prospective randomized trial. J Bone Joint Surg 2009; 91A (9):2104-12.

[13] Jacobs WC, Christen B, Wymenga AB, et al. Functional performance of mobile versus fixed bearing total knee prostheses: a randomised controlled trial. Knee Surg Sports Traumatol Arthrosc 2012; 20(8): 1450-55.

[14] Van der Bracht H, Van Maele G, Verdonk P, Almquist KF, Verdonk R, Freeman M. Is there any superiority in the clinical outcome of mobile-bearing knee prosthesis designs compared to fixed-bearing total knee designs in the treatment of osteoarthritis of the knee joint. Knee Surg Sports Traumatol Arthrosc 2010; 18: 367-74.
[15] Kalisvaart MM, Pagnano M, Trousdale RT, Stuart MJ, Hanssen AD. Randomized Clinical Trial of Rotating-Platform and FixedBearing Total Knee Arthroplasty: No Clinically Detectable Differences at Five Years. J Bone Joint Surg Am 2012; 94: 481-9.

[16] Kim Y-H, Yoon S-H, Kim J-S. The long term results of simultaneous fixed-bearing and mobile-bearing total knee replacements performed in the same patient. J Bone Joint Surg Br 2007; 89-B: 1317-23.

[17] Läderman A, Lübbeke A, Stern R, Riand N, Fritschy D. Fixedbearing versus mobile-bearing arthroplasty: A prospective randomised, clinical and radiological study with mid-term results at 7 years. Knee 2008; 15: 206-10.

[18] Wen Y, Liu D, Huang Y, Li B. A meta-analysis of the fixedbearing and mobile-bearing prostheses in total knee arthroplasty. Arch Orthop Trauma Surg 2011; 131: 1341-50.

(C) H.P. Delport; Licensee Bentham Open.

This is an open access article licensed under the terms of the Creative Commons Attribution Non-Commercial License (http://creativecommons.org/licenses/by-nc/3.0/) which permits unrestricted, non-commercial use, distribution and reproduction in any medium, provided the work is properly cited. 